\title{
Validation of a severity-of-illness score in patients with tuberculosis requiring intensive care unit admission
}

\author{
C F N Koegelenberg, ${ }^{1} \mathrm{MB}$ ChB, MMed (Int), FCP (SA), FRCP (UK), Cert Pulm (SA), PhD; C A Balkema, ${ }^{1} \mathrm{MD} ;$ Y Jooste, ${ }^{1} \mathrm{MB}$ ChB; \\ J J Taljaard, ${ }^{2} \mathrm{MB} \mathrm{ChB}$, MMed (Int); E M Irusen, ${ }^{1} \mathrm{MB} \mathrm{ChB}, \mathrm{FCP}(\mathrm{SA}), \mathrm{PhD}$ \\ ${ }^{1}$ Division of Pulmonology, Department of Medicine, Faculty of Medicine and Health Sciences, Stellenbosch University and Tygerberg \\ Academic Hospital, Tygerberg, Cape Town, South Africa \\ ${ }^{2}$ Division of Infectious Diseases, Department of Medicine, Faculty of Medicine and Health Sciences, Stellenbosch University and Tygerberg \\ Academic Hospital, Tygerberg, Cape Town, South Africa
}

Corresponding author: C F N Koegelenberg (coeniefn@sun.ac.za)

\begin{abstract}
Background. There is a paucity of data on the determinants of mortality due to tuberculosis (TB) in the intensive care unit (ICU). Objective. To develop a simple severity-of-illness score for use in patients with TB admitted to an ICU.

Methods. A scoring system was generated by retrospectively identifying the four most significant and clinically unrelated predictors of mortality from an existing prospectively collected dataset (January 2012 - May 2013), and combining these with known predictors of poor outcome.

Results. Of 83 patients admitted with TB, 38 (45.8\%) died in the ICU. The four parameters identified from the retrospective analysis were: (i) HIV co-infection with a CD4 cell count $<200 / \mu \mathrm{L}$; (ii) a raised creatinine level: (iii) a chest radiograph showing diffuse parenchymal infiltrates/miliary pattern; and (iv) absence of TB treatment on admission. These were combined with septic shock and a low arterial partial pressure of oxygen/fractional inspired oxygen (P:F) ratio to generate a six-point severity-of-illness score (one point for each parameter). The scores for survivors were significantly lower than those for non-survivors (mean (standard deviation) 2.27 (1.47) v. 3.58 (1.08); $p<0.01$ ). A score of $\geq 2$ was associated with significantly higher mortality than a score of $<2$ ( $7.1 \% \mathrm{v}$. $46.4 \%$; odds ratio (OR) 15.03 ; $95 \%$ confidence interval (CI) $1.86-121.32 ; p<0.01)$, whereas a score of $\geq 3$ was associated with a significantly higher mortality than a score of $<3(64.6 \%$ v. $20.0 \%$; OR 7.29 ; $95 \%$ CI $2.64-20.18 ; p<0.01$.

Conclusion. The proposed scoring system identified patients at increased risk of dying from TB in the ICU. Further prospective studies are indicated to validate its use.
\end{abstract}

S Afr Med J 2015;105(5):389-392. DOI:10.7196/SAMJ.9148

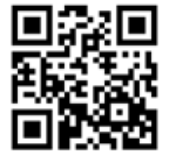

The incidence of tuberculosis (TB) remains high in much of the developing world, where infectious diseases including HIV still represent major challenges to healthcare systems. ${ }^{[1,2]}$ Previous studies have shown that approximately $1.5 \%$ of adults being treated for active $\mathrm{TB}$ in academic hospitals develop respiratory failure requiring admission to an intensive care unit (ICU). ${ }^{[3]}$

The mortality rate of patients with acute respiratory failure due to $\mathrm{TB}$ is higher than that of patients with respiratory failure due to other causes, and ranges from $40 \%$ to $80 \% .^{[2,4-6]}$ There is still a paucity of data on the determinants of mortality from TB in the ICU. Recent evidence suggests that the Acute Physiology and Chronic Health Evaluation II (APACHE II) score is predictive of patient mortality. ${ }^{[2]}$ The APACHE II score needs to be performed after 24 hours of ICU admission, however, and is not validated as a severity-of-illness score in an emergency setting. ${ }^{[7]}$

The aim of this study was to develop and retrospectively validate a simple severity-of-illness score for use in patients with TB requiring ICU admission.

\section{Methods}

\section{Study design and population}

We used data from a previously published study in which all patients with TB admitted to the medical ICU of Tygerberg Academic Hospital, Cape Town, South Africa (SA), from January 2012 to May 2013 were prospectively enrolled in order to identify potential parameters of poor outcome. ${ }^{[2]}$ A simple severity-of-illness score, based on our published dataset as well as other known poor prognostic factors, was subsequently retrospectively applied to the study population's admission data in order to assess its validity.
Tygerberg Academic Hospital, a 1380 -bed facility, is one of two referral centres in Cape Town and renders a tertiary service to a population of about 1.5 million. The study was approved by the Stellenbosch University Health Research Ethics Committee.

Patients were considered to have active TB if at least two of the following criteria were met: (i) smear positive for acid-fast bacilli (AFB) or GeneXpert MTB/RIF (Cepheid, SA) on sputum, tracheal aspirate or any other clinical specimen; (ii) culture positive for Mycobacterium tuberculosis (MTb) on sputum, tracheal aspirate or any other clinical specimen; (iii) histopathological identification of TB granuloma on biopsied tissues; (iv) strong clinical suspicion of active TB; ( $v$ ) strong radiological evidence of active TB; or ( $v i$ ) pleural fluid with a lymphocyte predominance ( $>75 \%$ lymphocytes and/or lymphocyte/neutrophil ratio $>0.75$ ) with an adenosine deaminase level $>40$ IU/L. A strong clinical suspicion of active TB required at least two of four constitutional symptoms (loss of weight with accompanying fever, night sweats, productive cough, loss of appetite for $>2$ weeks) as well as known TB contact or a history of previous pulmonary TB. Positive cultures were identified as MTb and tested for susceptibility to rifampicin and isoniazid using the MTBDRplus line probe assay (Hain LifeSciences, Germany).

\section{Clinical data, laboratory, imaging and related investigations}

Patient demographics, comorbid diseases (including HIV infection, AIDS, chronic obstructive pulmonary disease (COPD) and diabetes) and relevant medication use were documented.

Laboratory investigations collected included white cell count, platelet count, serum haemoglobin, serum albumin, C-reactive 
protein, creatinine, alanine aminotransferase (ALT) and serum glucose. Absolute CD4 counts were measured in all HIV-positive patients. All admission chest radiographs were reviewed by two pulmonologists blinded to the clinical data and classified as follows: (i) cavitation; (ii) miliary or diffuse interstitial infiltrates; (iii) lobar consolidation; (iv) pleural effusion; $(v)$ isolated lymphadenopathy; or $(v i)$ normal. The APACHE II score was calculated for all patients during the first 24 hours of the ICU stay. ${ }^{[7]}$

\section{Management, course and complications}

All patients were managed according to local guidelines and received maximal supportive therapy. ${ }^{[2,8]}$ The standard combination antiTB treatment regimen was used unless significant renal or hepatic impairment or confirmed drug resistance was present. ${ }^{[8]}$ Standard diagnostic criteria for the diagnosis of shock, renal failure, multiorgan dysfunction syndrome (MODS) and acute respiratory distress syndrome (ARDS) were used, as defined by accepted international criteria. ${ }^{[9-11]}$ Patients were categorised as either ICU/hospital survivors or non-survivors.

\section{Statistical aspects and development of the severity-of- illness score}

Descriptive statistics and $\chi^{2}$ or Fisher's exact tests (where indicated) were performed on dichotomous categorical variables, and $t$-tests on continuous data. A scoring system was generated by identifying the four most significant and clinically unrelated parameters identified from the prospective data collection and combining these with two known predictors of poor outcome: septic shock and a low arterial partial pressure of oxygen/fractional inspired oxygen (P:F) ratio..$^{[9,11]}$ For simplicity, we decided not to generate a weighted scoring system.

\section{Results}

\section{Study population characteristics}

Over the study period, 83 patients with TB (mean (standard deviation (SD)) age 36.5 (12.9) years, 38 males, 44 HIV-positive) were admitted to the medical ICU. The majority of the patients $(n=69,83.1 \%)$ had active pulmonary TB; 14 patients had exclusive evidence of extrapulmonary $\mathrm{TB}$ and 23 had both pulmonary and extrapulmonary TB. Extrapulmonary involvement included pleural disease $(n=13)$, disseminated disease $(n=11)$, meningitis $(n=7)$, abdominal TB $(n=4)$ and disease at other sites $(n=2)$. Thirty-two patients $(38.6 \%)$ were on TB treatment at the time of admission.

The mean duration of ICU admission was 11.9 days (range 1 - 56). The primary reason for admission was acute respiratory failure in two-thirds of patients $(n=56)$. Other reasons included a decreased level of consciousness $(n=7)$, surgery of the gastrointestinal tract $(n=3)$ and concomitant disease not directly related to TB $(n=3)$.

Tracheal aspirates (TAs) were obtained from all but three patients; 39.8\% were AFB-positive. Mycobacterial cultures obtained from a variety of sites, including TAs, were positive in 48 patients $(57.8 \%)$. Other sources included pleural fluid, cerebrospinal fluid (CSF), blood, lymph nodes, ascites, urine and placenta. The diagnosis of TB in the absence of direct microbiological proof was based on a high clinical probability combined with radiological evidence $(n=14)$, pleural fluid analysis alone $(n=5)$, CSF analysis $(n=7)$ or histological findings $(n=1)$. Only two patients had isoniazid monoresistance; none had multidrug-resistant TB (MDR-TB).

In total, 45 of 83 patients survived ICU admission (ICU mortality $45.8 \%$ ) and 34 survived to hospital discharge (in-hospital mortality $59.0 \%$ ). Three patients who were readmitted all survived their respective ICU and hospital admissions.

\section{Predictors of mortality}

The clinical, radiological and laboratory data of ICU survivors and non-survivors are summarised in Tables 1 - 3. Of the 31 patients with HIV co-infection and a CD4 count $<200$ cells/ $\mu \mathrm{L}, 16$ did not survive the ICU (OR 5.87; 95\% CI 1.11 - 30.95; $p=0.04$ ).

Renal failure was present in 31 patients. Of these, 24 did not survive hospital admission (OR 3.70; 95\% CI 1.15 - 10.09; $p=0.02$ ).

Neither ARDS $(n=26,35.6 \%)$ nor MODS $(n=25,30.1 \%)$ was associated with increased mortality. The ICU course was complicated by the development of ventilator-associated pneumonia in 19 patients (27.7\%), which did not adversely influence outcome.

\section{Suggested severity-of-illness score}

We combined septic shock and a low P:F ratio with the following four parameters identified as potentially significant in our study cohort: (i) HIV with CD4 count $<200 \mu \mathrm{L}$; (ii) renal failure; (iii) diffuse parenchymal infiltrates/miliary pattern on the chest radiograph; and (iv) absence of TB treatment on admission. We subsequently

\section{Table 1. Clinical data, ICU survivors v. non-survivor}

\begin{tabular}{|c|c|c|c|c|c|}
\hline & All $(N=83)$ & Survivors $(N=45)$ & Non-survivors $(N=38)$ & OR $(95 \% C I)$ & $p$-value \\
\hline Gender (female), $n$ & 45 & 25 & 20 & $0.89(0.37-2.11)$ & 1 \\
\hline Age (years), mean (SD) & $36.5(12.9)$ & $35.2(11.6)$ & $37.3(13.8)$ & N/A & 0.80 \\
\hline APACHE II, mean (SD) & $20.7(8.3)$ & $19.3(7.7)$ & $22.4(8.8)$ & $\mathrm{N} / \mathrm{A}$ & 0.09 \\
\hline Pulmonary TB, $n$ & 69 & 36 & 33 & $1.65(0.58-6.10)$ & 0.59 \\
\hline Isolated pulmonary TB, $n$ & 46 & 23 & 23 & $1.47(0.61-3.52)$ & 0.52 \\
\hline Extrapulmonary TB, $n$ & 37 & 22 & 15 & $0.68(0.28-1.63)$ & 0.26 \\
\hline Isolated extrapulmonary TB, $n$ & 14 & 9 & 5 & $0.61(0.18-1.99)$ & 0.56 \\
\hline HIV-positive, $n$ & 44 & 26 & 18 & $0.66(0.28-1.57)$ & 0.47 \\
\hline HIV with CD $4<200, n$ & $31 / 44$ & $15 / 26$ & $16 / 18$ & $5.87(1.11-30.95)$ & 0.04 \\
\hline AIDS, $n$ & 27 & 13 & 14 & $1.46(0.57-3.61)$ & 0.59 \\
\hline Diabetes mellitus, $n$ & 9 & 5 & 4 & $0.94(0.23-3.79)$ & 1 \\
\hline COPD, $n$ & 6 & 2 & 4 & $2.52(0.44-14.64)$ & 0.41 \\
\hline TB treatment on admission, $n$ & 32 & 22 & 10 & $0.37(0.15-0.95)$ & 0.06 \\
\hline
\end{tabular}


Table 2. Radiological findings, ICU survivors v. non-survivors

\begin{tabular}{|c|c|c|c|c|c|}
\hline Parameter & All $(N=83), n$ & Survivors $(N=45), n$ & Non-survivors $(N=38), n$ & OR $(95 \% \mathrm{CI})$ & $p$-value \\
\hline Cavitation & 12 & 10 & 2 & $0.19(0.04-0.95)$ & 0.03 \\
\hline Miliary and diffuse interstitial & 47 & 14 & 33 & $14.61(4.71-45.36)$ & $<0.01$ \\
\hline Lobar consolidation & 16 & 15 & 1 & $0.05(0.01-0.43)$ & $<0.01$ \\
\hline Pleural effusion & 13 & 9 & 4 & $0.47(0.13-1.67)$ & 0.38 \\
\hline Lymph node enlargement alone & 1 & 1 & 0 & N/A & N/A \\
\hline Normal & 1 & 1 & 0 & N/A & N/A \\
\hline
\end{tabular}

Table 3. Laboratory data, ICU survivors v. non-survivors

\begin{tabular}{|c|c|c|c|c|c|}
\hline Parameter & Reference & $\begin{array}{l}\text { Total }(N=83) \\
\text { Mean }(\mathrm{SD})\end{array}$ & $\begin{array}{l}\text { ICU survivors }(n=45) \\
\text { Mean }(\mathrm{SD})\end{array}$ & $\begin{array}{l}\text { ICU non-survivors }(n=38) \\
\text { Mean (SD) }\end{array}$ & $p$-value \\
\hline CD4 count (cells $/ \mu \mathrm{L})$ & $600-1500$ & $160.2(163.6)$ & $198.5(180.7)$ & $104.8(119.1)$ & 0.06 \\
\hline P:F ratio & $>300$ & $215.2(113.2)$ & $220.0(115.5)$ & $209.4(111.8)$ & 0.67 \\
\hline White cell count $\left(\times 10^{9} / \mathrm{L}\right)$ & $4.0-11.0$ & $13.5(7.1)$ & $14.1(6.7)$ & $12.8(7.6)$ & 0.41 \\
\hline Platelet count $\left(\times 10^{9} / \mathrm{L}\right)$ & $150-400$ & $282.8(165.7)$ & $307.6(175.1)$ & $253.4(150.9)$ & 0.14 \\
\hline Haemoglobin (g/dL) & $12.0-15.0^{*}$ & $9.76(2.41)$ & $9.52(2.55)$ & $10.04(2.23)$ & 0.26 \\
\hline Serum albumin $(g / L)$ & $35-50$ & $25.1(6.3)$ & $25.1(7.0)$ & $25.1(5.5)$ & 0.98 \\
\hline C-reactive protein $(\mathrm{mg} / \mathrm{L})$ & $<5$ & $175.2(94.4)$ & $168.8(93.3)$ & $183.0(97.0)$ & 0.53 \\
\hline Creatinine $(\mu \mathrm{mol} / \mathrm{L})$ & $<90^{*}$ & $137.7(152.0)$ & $131.6(139.4)$ & $145.0(167.4)$ & 0.69 \\
\hline $\operatorname{ALT}(\mathrm{U} / \mathrm{L})$ & $5-40$ & $133.8(305.1)$ & $178.6(398.8)$ & $84.8(138.1)$ & 0.21 \\
\hline Serum glucose $(\mathrm{mmol} / \mathrm{L})$ & $4.4-6.1$ & $8.80(4.76)$ & $8.32(4.55)$ & $9.35(5.00)$ & 0.33 \\
\hline
\end{tabular}

proposed the following scoring system, designated 'SCCOR-TB', with one point for each of the parameters: (i) septic shock; (ii) HIV with $\underline{\mathrm{CD}} 4<200 / \mu \mathrm{L}$; (iii) creatinine $>140 \mu \mathrm{mol} / \mathrm{L}$ (male) or $>120$ $\mu \mathrm{mol} / \mathrm{L}$ (female); (iv) P:F $\underline{\mathrm{O}}_{2}$ ratio $<200$; (v) chest radiograph showing diffuse parenchymal infiltrates/miliary pattern; and ( $v i)$ absence of TB treatment on admission. The scoring system was subsequently retrospectively applied to the study population's admission data.

\section{Mortality rates according to the suggested severity-of-illness score}

Severity-of-illness scores (Table 4) were significantly lower in survivors than in non-survivors (mean (SD) 2.27 (1.47) v. 3.58 (1.08); $p<0.01)$. Moreover, a score of $\geq 2$ was associated with a significantly higher mortality than a score of $<2(7.1 \%$ v. $46.4 \%$; OR $15.03 ; 95 \%$ CI 1.86 - 121.32; $p<0.01)$. Likewise, a score of $\geq 3$ was associated with a significantly higher mortality than a score of $<3(64.6 \%$ v. $20.0 \%$; OR 7.29; 95\% CI 2.64 - 20.18; $p<0.01$ ).

\section{Discussion}

We found the mortality rate among patients admitted to the ICU with $\mathrm{TB}$ to be extremely high: approximately $46 \%$ did not survive the ICU admission, and 59\% died in hospital. Although only a few clinical parameters, special investigations or other ancillary tests predicted outcome, we were able to retrospectively validate a simple sixpoint scoring system based on septic shock, HIV with CD4 counts, creatinine values, P:F ratios, chest radiography and TB treatment on admission. Severity-of-illness scores were significantly lower in survivors than non-survivors. Almost $1 / 2$ patients with a score of $\geq 2$ did not survive (v. $<1 / 10$ with a score of $<2 ; p<0.01$ ), whereas $2 / 3$ patients with a score of $\geq 3$ died (v. $1 / 5$ with a score of $<3$; $p<0.01$ ).
Table 4. Mortality rates according to the suggested severity-ofillness score

\begin{tabular}{lllll}
\hline SCCOR-TB & $\begin{array}{l}\text { Total } \\
(\boldsymbol{N = 8 3}), \boldsymbol{n}\end{array}$ & $\begin{array}{l}\text { Survivors } \\
(\boldsymbol{N}=\mathbf{4 5}), \boldsymbol{n}\end{array}$ & $\begin{array}{l}\text { Non-survivors } \\
(\boldsymbol{N}=\mathbf{3 8}), \boldsymbol{n}\end{array}$ & $\begin{array}{l}\text { Mortality, } \\
\%\end{array}$ \\
\hline$\leq 1$ & 14 & 13 & 1 & 7.1 \\
2 & 21 & 15 & 6 & 28.6 \\
3 & 18 & 10 & 8 & 44.4 \\
$\geq 4$ & 30 & 7 & 23 & 76.7 \\
$<2$ & 14 & 13 & 1 & 7.1 \\
$\geq 2$ & 69 & 32 & 37 & 46.4 \\
$<3$ & 35 & 28 & 7 & 20.0 \\
$\geq 3$ & 48 & 17 & 31 & 64.6
\end{tabular}

Our mean APACHE II score of 20.7 predicted a mortality rate of $30-40 \%,{ }^{[7]}$ suggesting that this globally used score underestimates mortality in this population. Moreover, it is only calculated after 24 hours of admission, making it an impractical initial severity-of-illness score for use in patients with TB requiring ICU admission. Simple severity-of-illness scores have been shown to be accurate in predicting a poor outcome in other severe illnesses, e.g. the Severity-of-Illness Score for Toxic Epidermal Necrolysis (SCORTEN), which was recently validated at our institution. ${ }^{[12]}$ Our data suggest that SCCOR-TB cutoffs of both $\geq 2$ and $\geq 3$ were associated with significant increments in mortality, which could potentially guide clinicians in identifying patients at a significantly increased risk of dying from TB in the ICU. 
We specifically aimed to identify parameters that could be readily assessed at practically all levels of medical care, and used both the prospective data set in addition to known poor prognostic factors (e.g. septic shock) to develop the score. ${ }^{[9]}$ Although the ORs of the various parameters differed, we opted to test our proposed scoring system with equally weighted parameters, in line with recent trends for simplicity. ${ }^{[13]}$ In an attempt not to 'double-penalise' HIV-positive patients and use clinically related parameters, we opted to include the parameter HIV with a CD4 count $<200 / \mu \mathrm{L}$ (OR 5.87), rather than AIDS (OR 1.46) or any other AIDS-defining diseases. Active TB itself has a significant impact on CD4 cell homeostasis, ${ }^{[14]}$ and our data suggest that non-survivors tend to have a lower count (105 v. 199; $p=0.06$ ).

We confirmed the well-known association between the lack of radiological evidence of cavitation and increased mortality. ${ }^{[15]}$ Moreover, we found that the presence of miliary and diffuse interstitial infiltrates was significantly associated with mortality (OR 14.6; $p<0.01)$. The latter parameter was therefore included in the proposed scoring system.

Delay in the initiation of anti-TB treatment is known to increase mortality. ${ }^{[16]}$ We found that survivors were more likely to be on TB treatment on admission than non-survivors $(p=0.06)$, and therefore also included this in our scoring system.

Our study has several potential limitations. We avoided suggesting a complex weighted scoring system (which would arguably have yielded a statistically more accurate system), as we aimed to develop a simple severity-of-illness score that can potentially be used at practically any level of care in SA. There was not a single case of MDR-TB in our cohort, and drug susceptibility was therefore not included in the scoring system. Furthermore, a selection bias towards healthier patients being referred and admitted to the ICU may have been present, given the limited ICU resources in our setting. ${ }^{[2]}$ We included a small proportion of cases (14/83) in which diagnoses were never microbiologically confirmed (diagnosed on clinical and radiological criteria). Although this is not an infrequent practice in studies from high endemic areas, ${ }^{[17]}$ it may have introduced some bias.

In conclusion, we were able to validate a simple scoring system based on six parameters: septic shock, HIV with CD4 counts, creatinine values, P:F ratios, chest radiography and TB treatment on admission. The proposed scoring system can potentially identify patients at an increased risk of dying from TB in the ICU. Further prospective studies are indicated to validate its use.

\section{Funding. None.}

\section{References}

1. World Health Organization. Global Tuberculosis Report 2013. Geneva: World Health Organization, 2013. http://www.who.int/tb/publications/global_report/en/ (accessed 10 November 2014).

2. Balkema C, Irusen E, Taljaard J, Koegelenberg C. Tuberculosis in the intensive care unit: A prospective observational study. Int J Tuberc Lung Dis 2014;18(7):824-830. [http://dx.doi.org/10.5588/ iitld.13.0044]

3. Levy H, Kallenbach J, Feldman C, Thorburn J, Abramowitz J. Acute respiratory failure in active tuberculosis. Crit Care Med 1987;15(3):221-225. [http://dx.doi.org/10.1097/00003246-19870300000008]

4. Lee PL, Jerng J, Chang Y, et al. Patient mortality of active pulmonary tuberculosis requiring mechanical ventilation. Eur Respir J 2003;22(1):141-147. [http://dx.doi.org/10.1183/09031936.03. 00038703]

5. Penner C, Roberts D, Kunimoto D, Manfreda J, Long R. Tuberculosis as a primary cause of respiratory failure requiring mechanical ventilation. Am J Respir Crit Care Med 1995;151(3):867-872. [http:// dx.doi.org/10.1164/ajrccm.151.3.7881684]

6. Zahar J, Azoulay E, Klement E, et al. Delayed treatment contributes to mortality in ICU patients with severe active pulmonary tuberculosis and acute respiratory failure. Intensive Care Med 2001;27(3):513520. [http://dx.doi.org/10.1007/s001340000849]

7. Knaus W, Draper E, Wagner D, Zimmerman J. APACHE II: A severity of disease classification system. Crit Care Med 1985;13(10):818-829. [http://dx.doi.org/10.1097/00003246-198510000-00009]

8. Koegelenberg CFN, Nortje A, Lalla U, et al. The pharmacokinetics of enteral antituberculosis drugs in patients requiring intensive care. S Afr Med J 2013;103(6):394-398. [http://dx.doi.org/10.7196/samj.6344]

9. Bone R, Balk R, Cerra F, et al. Definitions for sepsis and organ failure and guidelines for the use of innovative therapies in apsis. The ACCP/SCCM Consensus Conference Committee the College of Chest Physicians/Society of Critical Care Medicine. Chest 1992;101(6):1644-1655. [http:// College of Chest Physicians/Society of
dx.doi.org/10.1378/chest.101.6.1644]

10. Bellomo R, Ronco C, Kellum J, Mehta R, Palevsky P. Acute renal failure - definition, outcome measures Bellomo R, Ronco C, Kellum J, Mehta R, Palevsky P. Acute renal failure - definition, outcome measures,
animal models, fluid therapy and information technology needs: The Second International Consensus animal models, fluid therapy and information technology needs: The Second International Consensus
Conference of the Acute Dialysis Quality Initiative (ADQI) Group. Crit Care 2004;8(4):R204-R212. [http://dx.doi.org/10.1186/cc2872]

11. ARDS Definition Task Force, Ranieri V, Rubenfeld G, Thompson BT, et al. Acute respiratory distress syndrome: The Berlin Definition. JAMA 2012;307(23):2526-2533. [http://dx.doi.org/10.1001/ jama.2012.5669]

12. Kannenberg SM, Koegelenberg CF, Jordaan HF, von Groote-Bidlingmaier F, Visser WI. Toxi epidermal necrolysis and Stevens-Johnson syndrome in South Africa?: A 3-year prospective study. Q J Med 2012;105(9):839-846. [http://dx.doi.org/10.1093/qjmed/hcs078]

13. Douma R, Mos I, Erkens P, et al. Performance of 4 clinical decision rules in the diagnostic management of acute pulmonary embolism: A prospective cohort study. Ann Intern Med 2011;154(11):709-718. [http://dx.doi.org/10.7326/0003-4819-154-11-201106070-00002]

14. Skogmar S, Schön T, Balcha T, et al. CD4 cell levels during treatment for tuberculosis (TB) in Ethiopian adults and clinical markers associated with CD4 lymphocytopenia. PLoS One 2013;8(12):e83270. adults and clinical markers associated with CD4 1 [http://dx.doi.org/10.1371/journal.pone.0083270]

15. Barnes P, Leedom J, Chan L, et al. Predictors of short-term prognosis in patients with pulmonary . Barnes P, Leedom J, Chan L, et al. Predictors of short-term prognosis in patients with $\mathrm{p}$
tuberculosis. J Infect Dis 1988;158(2):366-371. [http://dx.doi.org/10.1093/infdis/158.2.366]

16. Zahar J, Azoulay E, Klement E, et al. Delayed treatment contributes to mortality in ICU patients with severe active pulmonary tuberculosis and acute respiratory failure. Intensive Care Med 2001;27(3):513520. [http://dx.doi.org/10.1007/s001340000849] [PMID: 11355119]

17. Theron G, Peter J, Meldau R, et al. Accuracy and impact of Xpert MTB/RIF for the diagnosis of smear negative or sputum-scarce tuberculosis using bronchoalveolar lavage fluid. Thorax 2013;68(11):10431051. [http://dx.doi.org/10.1136/thoraxjnl-2013-203485

Accepted 18 March 2015. 